\title{
Some Properties on the Harmonic Index of Molecular Trees
}

\author{
Shaoqiang Liu and Jianxi Li \\ School of Mathematics and Statistics, Minnan Normal University, Zhangzhou, Fujian 363000, China \\ Correspondence should be addressed to Jianxi Li; ptjxli@hotmail.com
}

Received 4 October 2013; Accepted 4 December 2013; Published 29 January 2014

Academic Editors: A. J. Kearsley and X. Liu

Copyright (C) 2014 S. Liu and J. Li. This is an open access article distributed under the Creative Commons Attribution License, which permits unrestricted use, distribution, and reproduction in any medium, provided the original work is properly cited.

The harmonic index of a graph $G$ is defined as the sum of weights $2 /(d(u)+d(v))$ of all edges $u v$ of $G$, where $d(u)$ denotes the degree of the vertex $u$ in $G$. In this paper, some general properties of the harmonic index for molecular trees are explored. Moreover, the smallest and largest values of harmonic index for molecular trees with given pendent vertices are provided, respectively.

\section{Introduction}

Let $G$ be a simple graph with vertex set $V(G)$ and edge set $E(G)$. Its order is $|V(G)|$, denoted by $n$. Let $d(v)$ and $N(v)$ be the degree and the set of neighbors of $v \in V(G)$, respectively. The harmonic index of $G$ is defined in [1] as

$$
H(G)=\sum_{u v \in E(G)} \frac{2}{d(u)+d(v)},
$$

where the summation goes over all edges $u v$ of $G$. This index was extensively studied recently. For example, Zhong $[2,3]$ and Zhong and $\mathrm{Xu}[4]$ determined the minimum and maximum values of the harmonic index for simple connected graphs, trees, unicyclic graphs, and bicyclic graphs, respectively. Some upper and lower bounds on the harmonic index of a graph were obtained by Ilic [5]. Xu [6] and Deng et al. $[7,8]$ established some relationship between the harmonic index of a graph and its topological indices, such as Randić index, atom-bond connectivity index, chromatic number, and radius, respectively. Wu et al. [9] determined the graph with minimum harmonic index among all the graphs (or all triangle-free graphs) with minimum degree at least two. More information on the harmonic index of a graph can be found in [10].

The general sum-connectivity index of $G$ was proposed by Du et al. in [11] and defined as

$$
\chi_{\alpha}(G)=\sum_{u v \in E(G)}(d(u)+d(v))^{\alpha} .
$$

Clearly, $H(G)=2 \chi_{-1}(G)$. Du et al. [11] determined the maximum value and the corresponding extremal trees for the general sum-connectivity indices of trees for $\alpha<\alpha_{0}$, where $\alpha_{0}=-4.3586 \ldots$ is the unique root of the equation $\left(4^{\alpha}-5^{\alpha}\right) /\left(5^{\alpha}-6^{\alpha}\right)=3$. However, they did not consider the general sum-connectivity indices with $\alpha=-1$.

A molecular tree $T$ is a tree with maximum degree at most four. It models the skeleton of an acyclic molecule [12]. As far as we know, the mathematical properties of related indices for molecular trees have been studied extensively. For example, Gutman et al. [13, 14] determined the molecular trees with the first maximum, the second maximum, and the third maximum Randić indices, respectively. Du et al. [15] further determined the fourth maximum Randić index for molecular trees. Li et al. [16, 17] obtained the lower and upper bounds for the general Randić index $R_{-1}$ for molecular trees and determined the molecular tree with minimum general Randić index among molecular trees with given pendant vertices. The graphs with maximum and minimum sum-connectivity indices among molecular trees with given pendant vertices were determined in Xing et al. [18].

In this paper, we consider the similar problem of determining the graphs with maximum or minimum harmonic index for molecular trees. Some general properties of the harmonic index for molecular trees are explored. Moreover, the smallest and largest values of harmonic index for molecular trees with given pendent vertices are determined, respectively. 


\section{Properties of the Harmonic Index for Molecular Trees}

In this section, some general properties of the harmonic index for molecular trees are explored. Before this, some notations are needed. Let $\mathscr{M} \mathscr{T}(n)$ be the set of molecular trees of order $n$. For $T \in \mathscr{M T}(n)$, denote by $n_{i}$ the number of vertices with degree $i$ for $i=1,2,3,4$, and denote by $x_{i j}$ the number of edges in $T$ that connect vertices of degree $i$ and $j$, where $1 \leq$ $i \leq j \leq 4$. Obviously, $n_{1}$ is the number of pendant vertices. Note that $x_{11}=0$. Then we have

$$
\begin{gathered}
n_{1}+n_{2}+n_{3}+n_{4}=n, \\
n_{1}+2 n_{2}+3 n_{3}+4 n_{4}=2(n-1), \\
x_{12}+x_{13}+x_{14}=n_{1}, \\
x_{12}+2 x_{22}+x_{23}+x_{24}=2 n_{2}, \\
x_{13}+x_{23}+2 x_{33}+x_{34}=3 n_{3}, \\
x_{14}+x_{24}+x_{34}+2 x_{44}=4 n_{4}, \\
H(T)=\frac{2}{3} x_{12}+\frac{1}{2} x_{13}+\frac{2}{5} x_{14}+\frac{1}{2} x_{22}+\frac{2}{5} x_{23} \\
+\frac{1}{3} x_{24}+\frac{1}{3} x_{33}+\frac{2}{7} x_{34}+\frac{1}{4} x_{44} .
\end{gathered}
$$

Moreover, Gutman and Miljković in [14] established the following relations:

$$
\begin{aligned}
x_{14}= & \frac{2 n+2}{3}-\frac{4}{3} x_{12}-\frac{10}{9} x_{13}-\frac{2}{3} x_{22}-\frac{4}{9} x_{23} \\
& -\frac{1}{3} x_{24}-\frac{2}{9} x_{33}-\frac{1}{9} x_{34}, \\
x_{44}= & \frac{n-5}{3}+\frac{1}{3} x_{12}+\frac{1}{9} x_{13}-\frac{1}{3} x_{22}-\frac{5}{9} x_{23} \\
& -\frac{2}{3} x_{24}-\frac{7}{9} x_{33}-\frac{8}{9} x_{34} .
\end{aligned}
$$

Substituting these equations into (4), we have

$$
\begin{aligned}
H(T)= & \frac{7 n}{20}-\frac{3}{20}+\frac{13}{60} x_{12}+\frac{1}{12} x_{13}+\frac{3}{20} x_{22} \\
& +\frac{1}{12} x_{23}+\frac{1}{30} x_{24}+\frac{1}{20} x_{33}+\frac{2}{105} x_{34} .
\end{aligned}
$$

Now, let

$$
\begin{aligned}
\varphi(T)= & H(T)-\left(\frac{7 n}{20}-\frac{3}{20}\right) \\
= & \frac{13}{60} x_{12}+\frac{1}{12} x_{13}+\frac{3}{20} x_{22}+\frac{1}{12} x_{23}+\frac{1}{30} x_{24} \\
& +\frac{1}{20} x_{33}+\frac{2}{105} x_{34} .
\end{aligned}
$$

Note that from (3), we have $n_{2}=(1 / 2) x_{12}+x_{22}+(1 / 2) x_{23}+$ $(1 / 2) x_{24}$ and $n_{3}=(1 / 3) x_{13}+(1 / 3) x_{23}+(2 / 3) x_{33}+(1 / 3) x_{34}$. Then we have the following lemma.
Lemma 1. For any $T \in \mathscr{M} \mathscr{T}(n)$, if $n_{2}+n_{3} \geq 3$, then $\varphi(T) \geq$ $72 / 420$.

Proof. We consider the following two cases.

Case $1\left(n_{2}+n_{3}>3\right)$. Note that

$$
\begin{aligned}
\varphi(T)= & \frac{13}{60} x_{12}+\frac{1}{12} x_{13}+\frac{3}{20} x_{22}+\frac{1}{12} x_{23} \\
& +\frac{1}{30} x_{24}+\frac{1}{20} x_{33}+\frac{2}{105} x_{34} \\
= & \frac{13}{60} x_{12}+\frac{3}{40} \times\left(2 x_{22}\right)+\frac{1}{24} x_{23}+\frac{1}{30} x_{24} \\
& +\frac{1}{12} x_{13}+\frac{1}{24} x_{23}+\frac{1}{40} \times\left(2 x_{33}\right)+\frac{2}{105} x_{34} \\
\geq & 2 n_{2} \times \frac{1}{30}+3 n_{3} \times \frac{2}{105} \geq \frac{96}{420} \quad \text { since } n_{2}+n_{3}>3 .
\end{aligned}
$$

Thus $\varphi(T) \geq 96 / 420>72 / 420$.

Case $2\left(n_{2}+n_{3}=3\right)$. If $n_{2}=3$ and $n_{3}=0$, then

$$
\begin{aligned}
\varphi(T) & =\frac{13}{60} x_{12}+\frac{3}{20} x_{22}+\frac{1}{30} x_{24} \\
& =\frac{13}{60} x_{12}+\frac{3}{40} \times\left(2 x_{22}\right)+\frac{1}{30} x_{24} \geq 6 \times \frac{1}{30} \\
& =\frac{84}{420}>\frac{72}{420} \quad \text { since } x_{12}+2 x_{22}+x_{24}=6 .
\end{aligned}
$$

If $n_{2}=2$ and $n_{3}=1$, then

$$
\begin{aligned}
\varphi(T)= & \frac{13}{60} x_{12}+\frac{1}{12} x_{13}+\frac{3}{20} x_{22}+\frac{1}{12} x_{23} \\
& +\frac{1}{30} x_{24}+\frac{1}{20} x_{33}+\frac{2}{105} x_{34} \\
= & \frac{13}{60} x_{12}+\frac{3}{40} \times\left(2 x_{22}\right)+\frac{1}{24} x_{23}+\frac{1}{30} x_{24} \\
& +\frac{1}{12} x_{13}+\frac{1}{24} x_{23}+\frac{1}{40} \times\left(2 x_{33}\right)+\frac{2}{105} x_{34} \\
\geq & 4 \times \frac{1}{30}+3 \times \frac{2}{105}=\frac{80}{420}>\frac{72}{420},
\end{aligned}
$$

since $x_{12}+2 x_{22}+x_{23}+x_{24}=4$ and $x_{13}+x_{23}+2 x_{33}+x_{34}=3$. If $n_{2}=1$ and $n_{3}=2$, then

$$
\begin{aligned}
\varphi(T)= & \frac{13}{60} x_{12}+\frac{1}{12} x_{13}+\frac{3}{20} x_{22}+\frac{1}{12} x_{23} \\
& +\frac{1}{30} x_{24}+\frac{1}{20} x_{33}+\frac{2}{105} x_{34} \\
= & \frac{13}{60} x_{12}+\frac{3}{40} \times\left(2 x_{22}\right)+\frac{1}{24} x_{23}+\frac{1}{30} x_{24}+\frac{1}{12} x_{13}
\end{aligned}
$$


TABLE 1: Nine classes of molecular trees and $\varphi(T)<72 / 420$.

\begin{tabular}{|c|c|c|c|c|c|c|}
\hline Set & $n_{2}$ & $n_{3}$ & Nonzero $x_{i j}^{\prime} s$ value & $\varphi(T)$ & $k$ & $n$ \\
\hline $\mathscr{M}_{1} T(n)$ & 0 & 0 & & 0 & 2 & $n \geq 5$ \\
\hline $\mathscr{M}_{2} T(n)$ & 0 & 1 & $x_{34}=3$ & $24 / 420$ & 1 & $n \geq 13$ \\
\hline $\mathscr{M}_{3} T(n)$ & 1 & 0 & $x_{24}=2$ & $28 / 420$ & 0 & $n \geq 9$ \\
\hline $\mathscr{M}_{4} T(n)$ & 0 & 2 & $x_{34}=6$ & $48 / 420$ & 0 & $n \geq 21$ \\
\hline $\mathscr{M}_{5} T(n)$ & 0 & 1 & $x_{34}=2, x_{13}=1$ & $51 / 420$ & 1 & $n \geq 10$ \\
\hline $\mathscr{M}_{6} T(n)$ & 0 & 2 & $x_{34}=4, x_{33}=1$ & $53 / 420$ & 0 & $n \geq 18$ \\
\hline $\mathscr{M}_{7} T(n)$ & 1 & 1 & $x_{24}=2, x_{34}=3$ & $52 / 420$ & 2 & $n \geq 17$ \\
\hline $\mathscr{M}_{8} T(n)$ & 2 & 0 & $x_{24}=4$ & $56 / 420$ & 1 & $n \geq 13$ \\
\hline $\mathscr{M}_{9} T(n)$ & 1 & 1 & $x_{24}=1, x_{34}=2, x_{23}=1$ & $65 / 420$ & 2 & $n \geq 14$ \\
\hline
\end{tabular}

$$
\begin{array}{r}
+\frac{1}{24} x_{23}+\frac{1}{40} \times\left(2 x_{33}\right)+\frac{2}{105} x_{34} \\
\geq 2 \times \frac{1}{30}+6 \times \frac{2}{105}=\frac{76}{420}>\frac{72}{420},
\end{array}
$$

since $x_{12}+2 x_{22}+x_{23}+x_{24}=2$ and $x_{13}+x_{23}+2 x_{33}+x_{34}=6$.

If $n_{2}=0$ and $n_{3}=3$, then

$$
\begin{aligned}
\varphi(T) & =\frac{1}{12} x_{13}+\frac{1}{20} x_{33}+\frac{2}{105} x_{34} \\
& =\frac{1}{12} x_{13}+\frac{1}{40} \times\left(2 x_{33}\right)+\frac{2}{105} x_{34} \\
& \geq 9 \times \frac{2}{105}=\frac{72}{420}, \quad \text { since } x_{13}+2 x_{33}+x_{34}=9 .
\end{aligned}
$$

This completes the proof.

Lemma 2. For any $T \in \mathscr{M} \mathscr{T}(n)$, if $n_{2}+n_{3} \leq 2$, then $\varphi(T)<$ $72 / 420$.

Proof. If $n_{2}+n_{3} \leq 2$, then the graphically feasible combinations of $x_{12}, x_{13}, x_{22}, x_{23}, x_{24}, x_{33}$, and $x_{34}$ for which $\varphi(T)<72 / 420$ are listed in Table 1 , where $n \equiv k(\bmod 3)$ and corresponding nine classes of molecular trees are denoted by $\mathscr{M}_{i} \mathscr{T}(n)$ for $i=1,2, \ldots, 9$, respectively.

Note that the smaller $\varphi(T)$, the smaller $H(T)$. Then by Lemmas 1 and 2, we have the following properties for $\mathscr{M} \mathscr{T}(n)$.

Theorem 3. For $n \equiv 0(\bmod 3)$, if $T \in \mathscr{M} \mathscr{T}(n)$, then

(a) when $n \geq 9, H(T) \geq(7 n / 20)-(1 / 12)$, the equality holds if and only if $T \in \mathscr{M}_{3} T(n)$;

(b) when $n \geq 21$ and $T \notin \mathscr{M}_{3} T(n), H(T) \geq(7 n / 20)-$ $(1 / 28)$, the equality holds if and only if $T \in \mathscr{M}_{4} T(n)$;

(c) when $n \geq 21$ and $T \notin \mathscr{M}_{3} T(n) \cup \mathscr{M}_{4} T(n), H(T) \geq$ $(7 n / 20)-(1 / 42)$, the equality holds if and only if $T \in$ $\mathscr{M}_{6} T(n)$.

Theorem 4. For $n \equiv 1(\bmod 3)$, if $T \in \mathscr{M T}(n)$, then

(a) when $n \geq 13, H(T) \geq(7 n / 20)-(13 / 140)$, the equality holds if and only if $T \in \mathscr{M}_{2} T(n)$; (b) when $n \geq 13$ and $T \notin \mathscr{M}_{2} T(n), H(T) \geq$ $(7 n / 20)-(1 / 35)$, the equality holds if and only if $T \in \mathscr{M}_{5} T(n)($ when $n=10, H(T)=(7 n / 20)-(1 / 35)$, and $\left.T \in \mathscr{M}_{5} T(n)\right)$;

(c) when $n \geq 13$ and $T \notin \mathscr{M}_{2} T(n) \cup \mathscr{M}_{5} T(n), H(T) \geq$ $(7 n / 20)-(1 / 60)$, the equality holds if and only if $T \in$ $\mathscr{M}_{8} T(n)$.

Theorem 5. For $n \equiv 2(\bmod 3)$, if $T \in M \mathscr{T}(n)$, then

(a) when $n \geq 5, H(T) \geq(7 n / 20)-(3 / 20)$, the equality holds if and only if $T \in \mathscr{M}_{1} T(n)$;

(b) when $n \geq 17$ and $T \notin \mathscr{M}_{1} T(n), H(T) \geq(7 n / 20)-$ $(11 / 420)$, the equality holds if and only if $T \in \mathscr{M}_{7} T(n)$;

(c) when $n \geq 17$ and $T \notin \mathscr{M}_{1} T(n) \cup \mathscr{M}_{7} T(n), H(T) \geq$ $(7 n / 20)+(1 / 210)$, the equality holds if and only if $T \in$ $\mathscr{M}_{9} T(n)$.

In [5], Ilic deduced that by removing an edge with the minimal weight from a graph, where the weight of $e=u v$ is denoted by $2 /(d(v)+d(u))$, its harmonic index strictly decreases. For molecular tree $T$, by removing any pendent vertex from $T$, we have the following theorem:

Theorem 6. Let $T$ be a molecular tree of order $n \geq 3$, and let $v$ be a pendent vertex of $T$. Then one has $H(T-v)<H(T)$.

Proof. Let $e=u v \in E(T)$ be a pendent edge, where $d(v)=1$ and $d(u) \geq 2$. Now we consider the difference $\Delta=H(T)-$ $H(T-v)$ in the following three cases.

Case $1\left(d(u)=2\right.$ and $\left.N(u)=\left\{v_{1}, v\right\}\right)$. The result follows from

$$
\begin{aligned}
\Delta & =\frac{2}{d\left(v_{1}\right)+2}+\frac{2}{3}-\frac{2}{d\left(v_{1}\right)+1} \\
& =\frac{2}{3}-\frac{2}{\left(2+d\left(v_{1}\right)\right)\left(d\left(v_{1}\right)+1\right)} \\
& \geq \frac{2}{3}-\frac{2}{(2+1)(1+1)}=\frac{1}{3} .
\end{aligned}
$$


Case $2\left(d(u)=3\right.$ and $\left.N(u)=\left\{v_{1}, v_{2}, v\right\}\right)$. The result follows from

$$
\begin{aligned}
\Delta & =\frac{2}{d\left(v_{1}\right)+3}+\frac{2}{d\left(v_{2}\right)+3}+\frac{2}{4}-\frac{2}{d\left(v_{1}\right)+2}-\frac{2}{d\left(v_{2}\right)+2} \\
& =\frac{1}{2}-\frac{2}{\left(3+d\left(v_{1}\right)\right)\left(d\left(v_{1}\right)+2\right)}-\frac{2}{\left(3+d\left(v_{2}\right)\right)\left(d\left(v_{2}\right)+2\right)} \\
& \geq \frac{1}{2}-2 \frac{2}{(3+1)(2+1)}=\frac{1}{6} .
\end{aligned}
$$

Case $3\left(d(u)=4\right.$ and $\left.N(u)=\left\{v_{1}, v_{2}, v_{3}, v\right\}\right)$. The result follows from

$$
\begin{aligned}
\Delta= & \frac{2}{d\left(v_{1}\right)+4}+\frac{2}{d\left(v_{2}\right)+4}+\frac{2}{d\left(v_{3}\right)+4}+\frac{2}{5}-\frac{2}{d\left(v_{1}\right)+3} \\
& -\frac{2}{d\left(v_{2}\right)+3}-\frac{2}{d\left(v_{3}\right)+3} \\
= & \frac{2}{5}-\frac{2}{\left(3+d\left(v_{1}\right)\right)\left(d\left(v_{1}\right)+4\right)}-\frac{2}{\left(3+d\left(v_{2}\right)\right)\left(d\left(v_{2}\right)+4\right)} \\
& -\frac{2}{\left(3+d\left(v_{3}\right)\right)\left(d\left(v_{3}\right)+4\right)} \\
\geq & \frac{2}{5}-3 \frac{2}{(3+1)(4+1)}=\frac{1}{10} .
\end{aligned}
$$

The proof is completed.

Remark 7. Note that removing a pendent vertex is equal to removing an pendent edge. Thus Theorem 6 states that, for molecular trees, the removed pendent vertex may not be located at an edge with the minimal weight, which is illustrated by the following example. In Figure 1, the weight of $e_{1}$ and that of $e_{2}$ are $2 /\left(d(u)+d\left(v_{1}\right)\right)=2 /(1+3)=1 / 2$ and $2 /\left(d\left(v_{2}\right)+d\left(v_{3}\right)\right)=2 /(3+3)=1 / 3$, respectively. But $H\left(T-e_{1}\right)=H(T-u)<H(T)$.

\section{Smallest Values of Harmonic Index for Molecular Trees with Given Pendent Vertices}

In this section, the smallest values of harmonic index for molecular trees with given number of pendent vertices are determined.

Theorem 8. Let $T$ be a tree of order $n$ with $p$ pendant vertices, where $2 \leq p \leq n-2$. Then

$$
H(T) \geq \frac{2}{p+2}-\frac{4}{p+1}+\frac{n-p}{2}+\frac{5}{3},
$$

the equality holds if and only if $T \cong S_{n, p}$, where $S_{n, p}$ (shown in Figure 2) is a tree obtained by attaching $p-1$ pendent vertices to an end vertex of the path $P_{n-p+1}$.

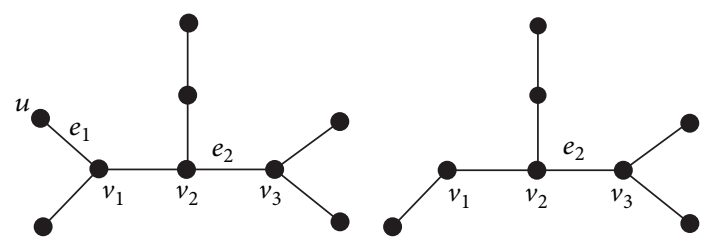

(a) $T, H(T)=56 / 15$

(b) $T-u, H(T-u)=52 / 15$

FIgURE 1: An example of $T-u$.

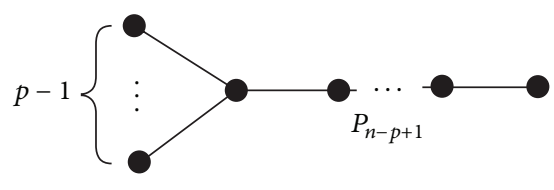

FIGURE 2: The tree $S_{n, p}$.

Proof. If $p=2$, then $T \cong P_{n}$; the result is obvious. For $3 \leq p \leq$ $n-2$, we prove the theorem by induction on $n$. If $n=5$, then $T \cong S_{5,3}$ or $T \cong S_{5}$; the result is obvious. Suppose that $n \geq 6$. Let $u$ be a pendant vertex and $N(u)=\{v\}$. Now we consider the $d(v)$ in the following two cases.

Case $1(d(v)=2)$. Then $T-u$ contains $p$ pendant vertices. For $n \geq 6$, there always exists $\omega \in N(v) \backslash\{u\}$ such that $d(w) \geq 2$. Thus

$$
\begin{aligned}
H(T)-H(T-u) & =\frac{2}{d(w)+2}+\frac{2}{3}-\frac{2}{d(w)+1} \\
& \geq \frac{2}{4}+\frac{2}{3}-\frac{2}{3}=\frac{1}{2} .
\end{aligned}
$$

The equality holds if and only if $d(w)=2$.

If $p=n-2$, then $T=S_{n, n-2}$; that is, $T-u=S_{n-1, n-2}$. Hence $d(w)>2$; if $3 \leq p \leq n-3$ and $T-u=S_{n-1, p}$, then $d(w)=2$. By the induction hypothesis, we have

$$
\begin{aligned}
H(T) & \geq H(T-u)+\frac{1}{2} \\
& \geq \frac{2}{p+2}+\frac{2(p-1)}{p+1}+\frac{2}{3}+\frac{2(n-p-3)}{4}+\frac{1}{2} \\
& =\frac{2}{p+2}-\frac{4}{p+1}+\frac{n-p}{2}+\frac{5}{3} .
\end{aligned}
$$

The equality holds if and only if $T-u=S_{n-1, p}$; that is, $T \cong S_{n, p}$. Case $2(d(v) \geq 3)$. Then $T-u$ contains $p-1$ pendant vertices, and $N(v) \backslash\{u\}$ contains some vertices with degree at least two. For $3 \leq p \leq n-2$, we have 


$$
\begin{aligned}
H(T)-H(T-u) \\
=\frac{2}{d(v)+1}-\sum_{w \in N(v) \backslash u}\left(\frac{2}{d(w)+d(v)-1}-\frac{2}{d(w)+d(v)}\right) \\
\geq \frac{2}{d(v)+1}-\left(\frac{2}{d(v)-1+2}-\frac{2}{d(v)+2}\right) \\
-(d(v)-2)\left(\frac{2}{d(v)-1+1}-\frac{2}{d(v)+1}\right) \\
=2\left(\frac{1}{d(v)+2}-\frac{3}{d(v)+1}+\frac{2}{d(v)}\right)
\end{aligned}
$$

The equality holds if and only if $N(v)$ contains one vertex of degree two and $d(v)-1$ vertices of degree one. Let $g(x)=$ $2((1 /(x+2))-(3 /(x+1))+(2 / x))$. Note that $g^{\prime}(x)=-\left(\left(\left(4 x^{3}+\right.\right.\right.$ $\left.\left.\left.30 x^{2}+48 x+16\right)\right) /\left(\left((x+2)^{2}(x+1)^{2} x^{2}\right)\right)\right)<0$ for $x \geq 0$. Then $g(x)$ is strictly decreasing on $x \geq 0$. Recall that $d(v) \leq p$. Hence we have

$$
H(T)-H(T-u) \geq 2\left(\frac{1}{p+2}-\frac{3}{p+1}+\frac{2}{p}\right) .
$$

The equality holds if and only if $N(v)$ contains one vertex of degree two and $d(v)-1$ vertices of degree one; that is, $T=S_{n, p}$ and $d(v)=p$. By the induction hypothesis, we have

$$
\begin{aligned}
H(T) & \geq H(T-u)+2\left(\frac{1}{p+2}-\frac{3}{p+1}+\frac{2}{p}\right) \\
& \geq \frac{2}{p+1}-\frac{4}{p}+\frac{n-p}{2}+\frac{5}{3}+2\left(\frac{1}{p+2}-\frac{3}{p+1}+\frac{2}{p}\right) \\
& =\frac{2}{p+2}-\frac{4}{p+1}+\frac{n-p}{2}+\frac{5}{3}
\end{aligned}
$$

The equality holds if and only if $T-u \cong S_{n-1, p-1}$ and $d(v)=p$; that is, $T \cong S_{n, p}$.

Lemma 9. Let $n, p$ be positive integers with $2 \leq p \leq n-2$. Let

$$
f(n, p)=\frac{2}{p+2}-\frac{4}{p+1}+\frac{n-p}{2}+\frac{5}{3}
$$

Then $f(n, p)$ is monotonically decreasing on $p$.

Proof. We consider the derivative of $f(n, p)$. For $p \geq 2$, we have

$$
\begin{aligned}
\frac{\partial f(n, p)}{\partial p} & =-\frac{2}{(p+2)^{2}}+\frac{4}{(p+1)^{2}}-\frac{1}{2} \\
& =\frac{-p^{4}-6 p^{3}-9 p^{2}+12 p+24}{2(p+1)^{2}(p+2)^{2}}
\end{aligned}
$$

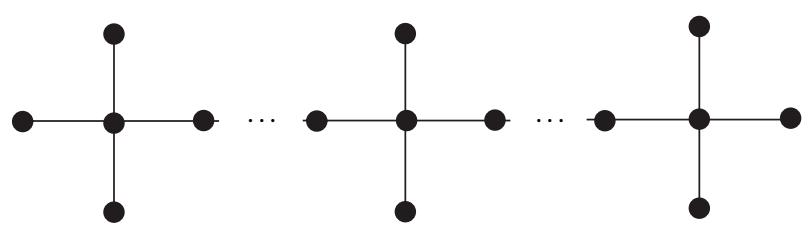

Figure 3: An example of molecular tree in $\mathscr{L}_{e}(n, p)$.

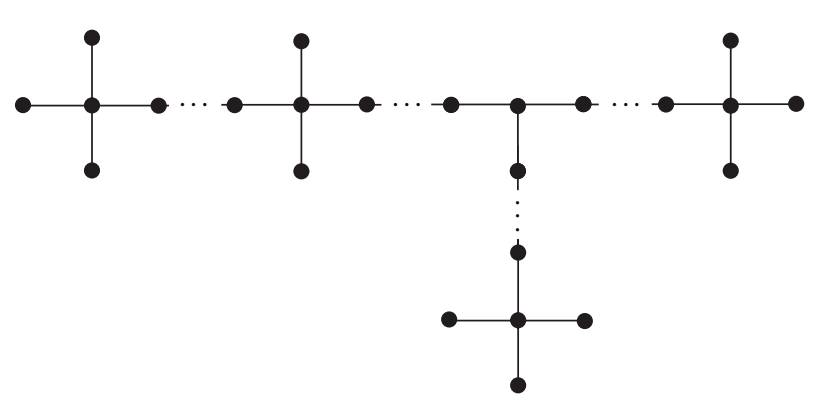

Figure 4: An example of molecular tree in $\mathscr{L}_{l}(n, p)$.

Let $g(p)=-p^{4}-6 p^{3}-9 p^{2}+12 p+24$. Clearly, $g(2)<0$. We consider the derivative of $g(p)$. For $p \geq 2$, we have

$$
g^{\prime}(p)=-4 p^{3}-18 p^{2}-18 p+12<0
$$

Thus $g(p)$ is monotonically decreasing on $p$ and $g(p) \leq$ $g(2)<0$. That is, $\partial f(n, p) / \partial p<0$ for $2 \leq p \leq n-2$. Hence $f(n, p)$ is monotonically decreasing on $p$.

Recall that if $p=n-1$, then $S_{n, n-1}=S_{n}$ and $H\left(S_{n}\right)=2(n-$ $1) / n$. Moreover, by Lemma 9 , we have $f(n, p) \geq f(n, n-2)=$ $(2 / n)-(4 /(n-1))+1+(5 / 3)>2(n-1) / n=H\left(S_{n}\right)$. This together with Theorem 8 implies the following

Corollary 10 (see [5]). Among all trees of order $n$, the minimum harmonic index is attained uniquely by the star $S_{n}$.

Let $\mathscr{M} \mathscr{T}_{n, p}$ be the set of molecular trees of order $n$ with $p$ pendent vertices. Now we introduce two classes of molecular trees of order $n$ with $p$ pendent vertices.

The first class is denoted by $\mathscr{L}_{e}(n, p)$ for even $p$ with $6 \leq$ $p \leq\lfloor(n+3) / 2\rfloor$ (shown in Figure 3). Those trees are composed of $(p-2) / 2$ star $S_{5}$, which are connected by paths whose lengths may be zero. Note that $n_{1}=p, n_{2}=n-(3 p / 2)+1, n_{3}=$ $0, n_{4}=(p / 2)-1, x_{14}=p, x_{22}=n-2 p+3$, and $x_{24}=p-4$.

The second class is denoted by $\mathscr{L}_{l}(n, p)$ for odd $p$ with $9 \leq$ $p \leq\lfloor(n+2) / 2\rfloor$ (shown in Figure 4$)$. Those trees are composed of $(p-3) / 2$ star $S_{5}$ and one star $S_{4}$, which are connected by paths whose lengths may be zero, and the unique star $S_{4}$ is connected by three stars $S_{5}$. Note that $n_{1}=p, n_{2}=n-((3 p+$ $1) / 2)+1, n_{3}=1, n_{4}=((p-1) / 2)-1, x_{14}=p, x_{24}=p-6$, $x_{22}=n-2 p+2$, and $x_{23}=3$.

For $p \leq 4$, if $3 \leq n \leq 5$, then Corollary 10 implies that $S_{n}$ is the unique molecular tree with the minimum harmonic index; if $n \geq 6$, then Theorem 8 and Lemma 9 imply that $S_{n, 4}$ is the unique molecular tree with the minimum harmonic index. If $p \geq 5$, then $S_{n, p}$ is not a molecular tree. The following gives the smallest value for $\mathscr{M T}_{n, p}$ with $p \geq 5$. 
Theorem 11. Let $T \in M \mathscr{T}_{n, p}$ and $p \geq 5$. Then

$$
H(T) \geq \frac{n}{2}-\frac{4}{15} p+\frac{1}{6} ;
$$

the equality holds if and only if $T \in \mathscr{L}_{e}(n, p)$ for even $p$ with $6 \leq p \leq\lfloor(n+3) / 2\rfloor$. Moreover, if $p$ is odd and $9 \leq p \leq$ $\lfloor(n+2) / 2\rfloor$, then

$$
H(T) \geq \frac{n}{2}-\frac{4}{15} p+\frac{1}{5} ;
$$

the equality holds if and only if $T \in \mathscr{L}_{l}(n, p)$.

Proof. For $T \in M \mathscr{T}_{n, p}$, Xing et al. [18] deduced the following relations:

$$
\begin{gathered}
x_{14}=p-x_{12}-x_{13}, \\
x_{22}=n-2 p-x_{12}-\frac{1}{3} x_{13}-\frac{1}{3} x_{23}+\frac{1}{3} x_{33}+\frac{2}{3} x_{34}+x_{44}+3, \\
x_{24}=p+x_{12}+\frac{1}{3} x_{13}-\frac{2}{3} x_{23}-\frac{4}{3} x_{33}-\frac{5}{3} x_{34}-2 x_{44}-4 .
\end{gathered}
$$

Substituting these equations into (4), we have

$$
\begin{aligned}
H(T)= & \frac{n}{2}-\frac{4}{15} p+\frac{1}{6}+\frac{1}{10} x_{12}+\frac{2}{45} x_{13}+\frac{1}{90} x_{23} \\
& +\frac{1}{18} x_{33}+\frac{4}{63} x_{34}+\frac{1}{12} x_{44} .
\end{aligned}
$$

Clearly, the minimum value of $H(T)$ is attained at $x_{12}=x_{13}=$ $x_{23}=x_{33}=x_{34}=x_{44}=0$. That is $H(T) \geq(n / 2)-(4 / 15) p+$ $(1 / 6)$. Moreover if $H(T)=(n / 2)-(4 / 15) p+(1 / 6)$, then $x_{14}=$ $p, x_{22}=n-2 p+3, x_{24}=p-4, n_{2}=n-(3 p / 2)+1, n_{3}=0$, and $n_{4}=(p / 2)-1$, implying that $T \in \mathscr{L}_{e}(n, p)$.

Now suppose that not all of $x_{12}, x_{13}, x_{23}, x_{33}, x_{34}$, and $x_{44}$ in (28) are zero. Let

$$
\begin{aligned}
\varphi(T) & =H(T)-\left(\frac{n}{2}-\frac{4}{15} p+\frac{1}{6}\right) \\
& =\frac{1}{10} x_{12}+\frac{2}{45} x_{13}+\frac{1}{90} x_{23}+\frac{1}{18} x_{33}+\frac{4}{63} x_{34}+\frac{1}{12} x_{44} .
\end{aligned}
$$

Now we consider the following two cases.

Case $1\left(x_{12}+x_{13}+x_{33}+x_{34}+x_{44}>0\right)$. Then $\varphi(T) \geq$ $\min \{(1 / 10),(2 / 45),(1 / 18),(4 / 63),(1 / 12)\}=(2 / 45)>(1 / 30)$, the result holds.

Case $2\left(x_{12}=x_{13}=x_{33}=x_{34}=x_{44}=0\right.$ and $\left.x_{23} \neq 0\right)$. Clearly, $x_{23}=3 n_{3}$ since $x_{13}+x_{23}+2 x_{33}+x_{34}=3 n_{3}$. Thus the only possible combination of $x_{12}, x_{13}, x_{23}, x_{33}, x_{34}$, and $x_{44}$ for which $\varphi(T) \leq(1 / 30)$ is $x_{12}=x_{13}=x_{33}=x_{34}=x_{44}=0$, $x_{23}=3$ with $n_{3}=1$.

Hence the minimum value of $H(T)$ is attained at $x_{12}=$ $x_{13}=x_{33}=x_{34}=x_{44}=0, x_{23}=3$. That is, $H(T) \geq(n / 2)-$ $(4 / 15) p+(1 / 5)$. Moreover if $H(T)=(n / 2)-(4 / 15) p+(1 / 5)$, then $x_{14}=p, x_{22}=n-2 p+2, x_{24}=p-6, n_{2}=n-((3 p+$ $1) / 2)+1, n_{3}=1$, and $n_{4}=((p-1) / 2)-1$, implying that $T \in \mathscr{L}_{l}(n, p)$.This completes the proof.

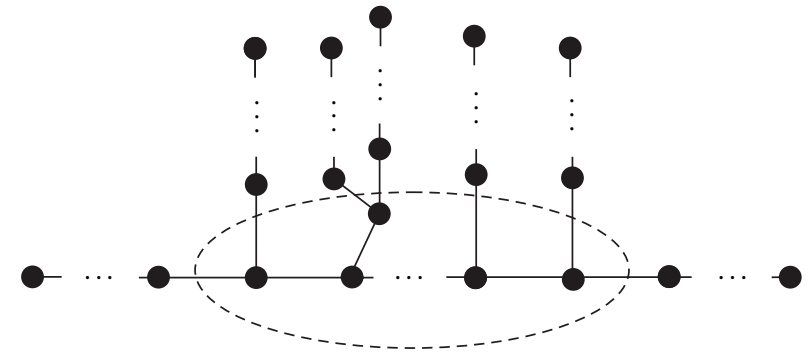

Figure 5: An example of $L_{3}(n, p)$.

\section{Largest Values for Harmonic Index of Molecular Trees with Given Pendent Vertices}

In this section, largest values for the harmonic indices of molecular trees with given number of pendent vertices are determined.

For $T \in M \mathscr{T}_{n, p}$, let $V_{i}(T)=\{v: v \in V(T), d(v)=i\}$ and $E_{i, j}(T)=\{e: e=u v \in E(T), d(u)=i, d(v)=j\}$.

Let $\mathscr{L}_{3}(n, p)$ (shown in Figure 5 ) be a class of molecular trees of order $n$. For this type of molecular trees, there are $p-2$ vertices of maximal degree three, which induce a tree and any of these vertices is adjacent to either another vertex of degree three or a vertex of degree two. Note that $n_{1}=p$, $n_{2}=n-2 p+2, n_{3}=p-2, n_{4}=0, x_{12}=x_{23}=p, x_{13}=0$, $x_{22}=n-3 p+2$, and $x_{33}=p-3$. Clearly, $3 \leq p \leq\lfloor(n+2) / 3\rfloor$.

Lemma 12. Let $T$ be a tree with maximum harmonic index among all trees in $\mathscr{M T}_{n, p}$. Then either $E_{22}(T)=\emptyset$ or $V_{4}(T) \bigcup E_{13}(T)=\emptyset$.

Proof. We shall prove the contrapositive of the lemma. If $E_{22}(T) \neq \emptyset$ and $V_{4}(T) \cup E_{13}(T) \neq \emptyset$, then either $E_{22}(T) \neq \emptyset$ and $V_{4}(T) \neq \emptyset$, or $E_{22}(T) \neq \emptyset$ and $E_{13}(T) \neq \emptyset$.

If $E_{22}(T) \neq \emptyset$ and $V_{4}(T) \neq \emptyset$, then there exists a vertex $v \in$ $V_{4}(T)$ and $N(v)$ has at most one neighbor of degree four. Let $T^{\prime}$ be the tree obtained from $T$ by contracting the edge $e \in$ $E_{22}(T)$ and splitting the vertex $v$ into $\left(v_{1}, v_{2}\right)$. Clearly, $T^{\prime} \in$ $\mathscr{M}_{n, p}$ and

$$
\begin{aligned}
& H\left(T^{\prime}\right)-H(T) \\
& =\frac{2}{3+3}-\frac{2}{2+2}+\sum_{w \in N(v)}\left(\frac{2}{3+d(w)}-\frac{2}{4+d(w)}\right) \\
& =-\frac{1}{6}+\sum_{w \in N(v)}\left(\frac{2}{3+d(w)}-\frac{2}{4+d(w)}\right) \\
& >-\frac{1}{6}+3\left(\frac{2}{3+3}-\frac{2}{4+3}\right)+\frac{2}{3+4}-\frac{2}{4+4}=\frac{1}{84}>0 ;
\end{aligned}
$$

if $E_{22}(T) \neq \emptyset$ and $E_{13}(T) \neq \emptyset$, suppose that $u v \in E_{13}(T)$, $d(u)=1$ and $d(v)=3$. Let $T^{\prime}$ be the tree obtained from $T$ 
by contracting the edge $e \in E_{22}(T)$ and attaching an edge to $u$. Clearly, $T^{\prime} \in \mathscr{M} \mathscr{T}_{n, p}$ and

$$
H\left(T^{\prime}\right)-H(T)=\frac{2}{2+1}+\frac{2}{2+3}-\frac{2}{2+2}-\frac{2}{1+3}=\frac{1}{15}>0 .
$$

For each case, we have $H\left(T^{\prime}\right)>H(T)$, which contradicts $T$ with maximum harmonic index among all trees in $\mathscr{M}_{n, p}$. This completes the proof.

Theorem 13. Let $T \in M \mathscr{T}_{n, p}$ with $p \geq 2$. Then

$$
H(T) \leq \frac{n}{2}-\frac{1}{12} p
$$

the equality holds if and only if $T \cong P_{n}$.

Proof. For any molecular tree $T$, the following relations were deduced by Xing et al. in [18]:

$$
\begin{gathered}
x_{12}=p-x_{13}-x_{14}, \\
x_{22}=n-\frac{5}{2} p+\frac{1}{2} x_{13}+\frac{3}{4} x_{14}-\frac{1}{2} x_{23}-\frac{1}{4} x_{24} \\
+\frac{1}{4} x_{34}+\frac{1}{2} x_{44}+2, \\
x_{33}=\frac{3}{2} p-\frac{1}{2} x_{13}-\frac{3}{4} x_{14}-\frac{1}{2} x_{23}-\frac{3}{4} x_{24} \\
-\frac{5}{4} x_{34}-\frac{3}{2} x_{44}-3 .
\end{gathered}
$$

Substituting these equations into (4), we have

$$
\begin{aligned}
H(T)= & \frac{n}{2}-\frac{1}{12} p-\frac{1}{12} x_{13}-\frac{17}{120} x_{14} \\
& -\frac{1}{60} x_{23}-\frac{1}{24} x_{24}-\frac{1}{168} x_{34} .
\end{aligned}
$$

Clearly, the maximum value of $H(T)$ is attained at $x_{13}=x_{14}=$ $x_{23}=x_{24}=x_{34}=0$. That is, $H(T) \geq(n / 2)-(1 / 12) p$. Moreover, if $H(T)=(n / 2)-(1 / 12) p$, then $x_{12}=p, x_{22}=$ $n-(5 / 2) p+2, x_{33}=(3 / 2) p-3$. Note that $x_{33}=x_{44}=0$ since $T \in \mathscr{M} \mathscr{T}_{n, p}$; that is, $p=2, x_{12}=2$, and $x_{22}=n-3$. This implies that $T \cong P_{n}$.

Theorem 14. Let $T \in \mathscr{M} \mathscr{T}_{n, p}\left(T \neq P_{n}\right)$ with $\left|E_{23}(T)\right|=p$ and $\left|E_{22}(T)\right| \neq \emptyset$. Then

$$
H(T) \leq \frac{n}{2}-\frac{1}{10} p ;
$$

the equality holds if and only if $T \in \mathscr{L}_{3}(n, p)$.

Proof. From the proof of Theorem 13, we can see that if $T \neq P_{n}$, then not all of $x_{13}, x_{14}, x_{23}, x_{24}$, and $x_{34}$ in (34) are zero. By Lemma 12, if $T$ with maximum harmonic index among all trees in $\mathscr{M} \mathscr{T}_{n, p}$ and $E_{22}(T) \neq \emptyset$, then $V_{4}(T) \bigcup E_{13}(T)=\emptyset$. That is, $x_{14}=x_{24}=x_{34}=x_{44}=x_{13}=0$. Theorem 13 implies that $x_{12}=p, x_{22}=n-(5 / 2) p-(1 / 2) x_{23}+2$, and $x_{33}=(3 / 2) p-(1 / 2) x_{23}-3$. If $\left|E_{23}(T)\right|=p$, then $x_{23}=p$, $x_{12}=p, x_{22}=n-3 p+2$, and $x_{33}=p-3$. Note that $p \geq 3$ and $\left|E_{23}(T)\right|=p$ since $T \neq P_{n}$. Then $H(T) \leq(2 p / 3)+(2 p /(2+3))+$ $((2(p-3)) /(3+3))+((2(n-3 p+2)) /(2+2))=(n / 2)-(1 / 10) p$; the equality holds if and only if $T \in \mathscr{L}_{3}(n, p)$. This completes the proof.

\section{Conflict of Interests}

The authors declare that there is no conflict of interests regarding the publication of this paper.

\section{Acknowledgments}

This work is partially supported by NSF of China (no. 11101358), NSF of Fujian (nos. 2011J05014 and 2011J01026), and Project of Fujian Education Department (no. JA11165).

\section{References}

[1] S. Fajtlowicz, "On conjectures of Graffiti-II," Congressional Number, vol. 60, pp. 187-197, 1987.

[2] L. Zhong, "The harmonic index for graphs," Applied Mathematics Letters, vol. 25, no. 3, pp. 561-566, 2012.

[3] L. Zhong, "The harmonic index on unicyclic graphs," Ars Combinatoria, vol. 104, pp. 261-269, 2012.

[4] L. Zhong and K. Xu, "The harmonic index for bicyclic graphs," Utilitas Mathematica, vol. 90, pp. 23-32, 2013.

[5] A. Ilic, "Note on the harmonic index of a graph," Applied Mathematics Letters, vol. 25, no. 3, pp. 561-566, 2012.

[6] X. Xu, "Relationships between harmonic index and other topological indices," Applied Mathematical Sciences, vol. 6, no. 41, pp. 2013-2018, 2012.

[7] H. Deng, Z. Tang, and J. Zhang, On the harmonic index and the radius of a graph, preprint.

[8] H. Deng, S. Balachandran, S. K. Ayyaswamy, and Y. B. Venkatakrishnan, "On the harmonic index and the chromatic number of a graph," Discrete Applied Mathematics, vol. 161, no. 16-17, pp. 2740-2744, 2013.

[9] R. Wu, Z. Tang, and H. Deng, "A lower bound for the harmonic index of a graph with minimum degree at least two," Filomat, vol. 27, no. 1, pp. 51-55, 2013.

[10] H. Deng, "A unified approach to the extremal Zagreb indices for trees, unicyclic graphs and bicyclic graphs," Match, vol. 57, no. 3, pp. 597-616, 2007.

[11] Z. Du, B. Zhou, and N. Trinajstic, "On the sum-connectivity index of trees," Applied Mathematics Letters, vol. 24, pp. 402405, 2011.

[12] N. Trinajstic, Chemical Graph Theory, CRC Press, Boca Raton, Fla, USA, 2nd edition, 1992.

[13] I. Gutman, O. Miljković, G. Caporossi, and P. Hansen, "Alkanes with small and large Randić connectivity indices," Chemical Physics Letters, vol. 306, no. 5-6, pp. 366-372, 1999.

[14] I. Gutman and O. Miljković, "Molecules with smallest connectivity indices," Match, vol. 41, pp. 57-70, 2000.

[15] Z. Du, B. Zhou, and N. Trinajstic, "Molecules with smallest connectivity indices," Communications in Mathematical and in Computer Chemistry, vol. 62, pp. 131-142, 2009.

[16] X. Li and Y. Yang, "Best lower and upper bounds for the Randić index R-1 of chemical trees," Match, no. 52, pp. 147-156, 2004. 
[17] X. Li, Y. Shi, and L. Zhong, "Minimum general Randić index on chemical trees with given order and number of pendent vertices," Match, vol. 60, no. 2, pp. 539-554, 2008.

[18] R. Xing, B. Zhou, and N. Trinajstić, "Sum-connectivity index of molecular trees," Journal of Mathematical Chemistry, vol. 48, no. 3, pp. 583-591, 2010. 


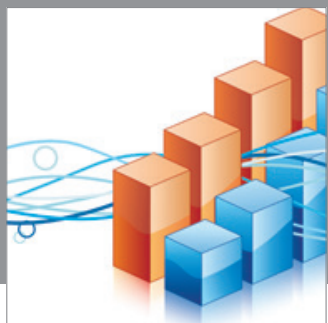

Advances in

Operations Research

mansans

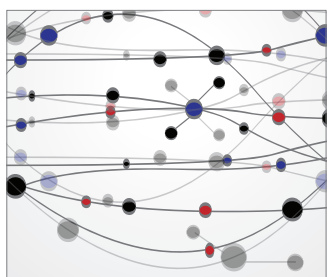

The Scientific World Journal
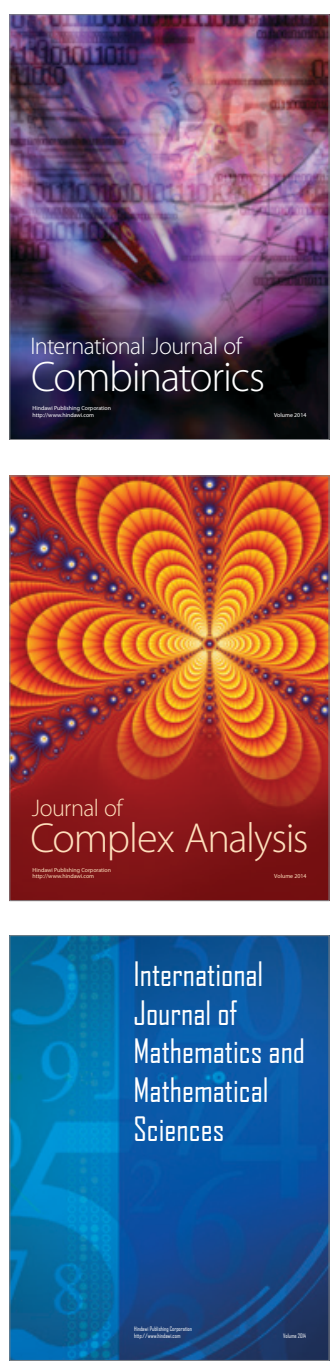
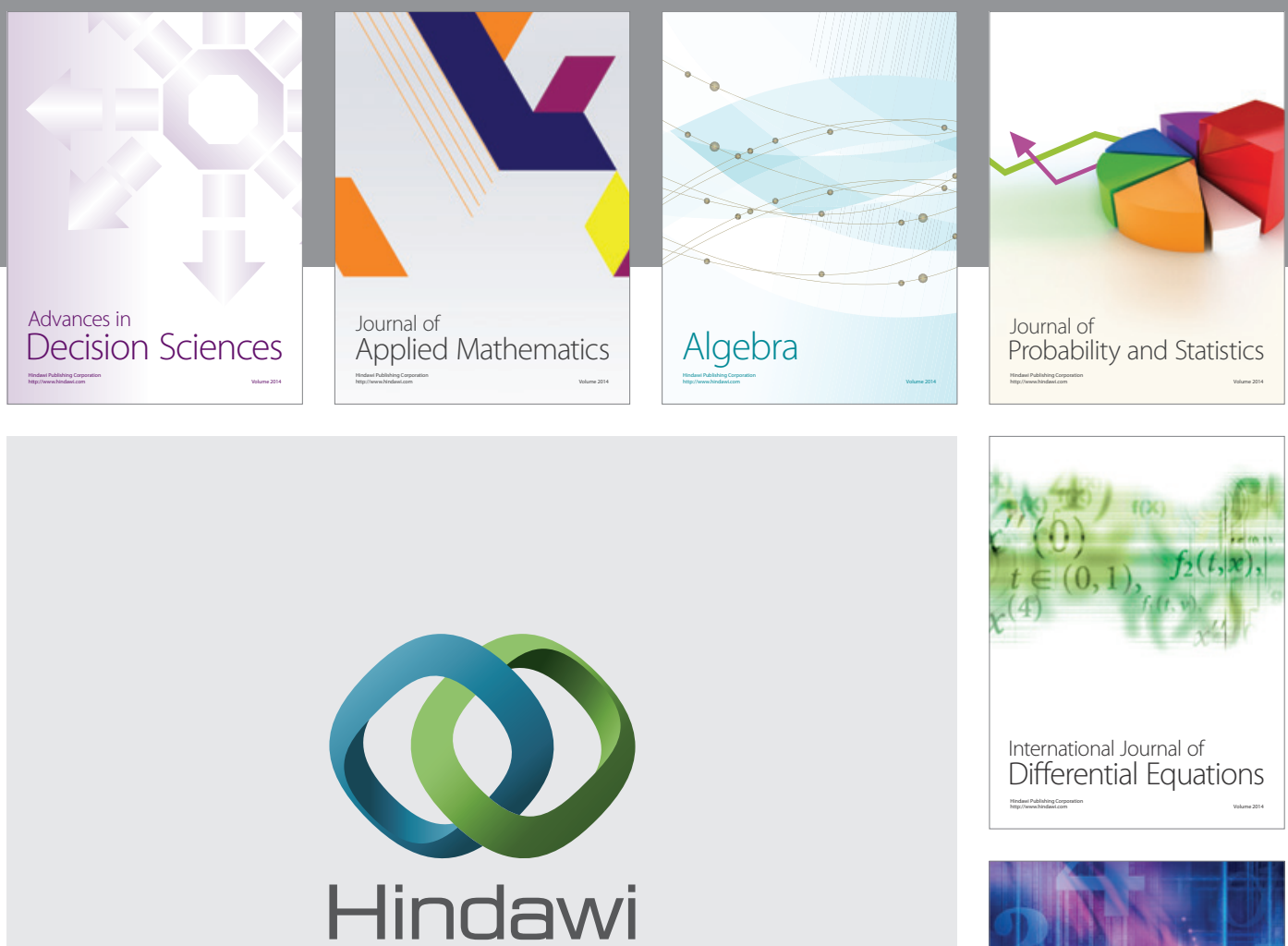

Submit your manuscripts at http://www.hindawi.com
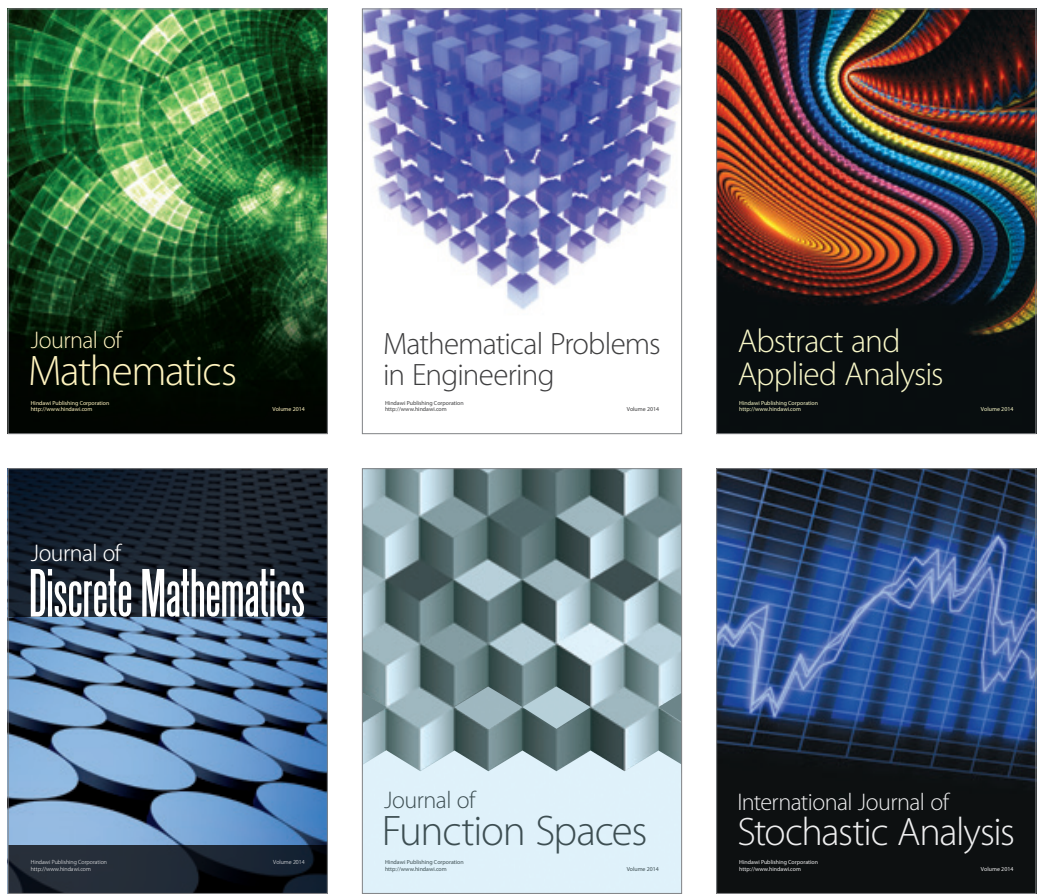

Journal of

Function Spaces

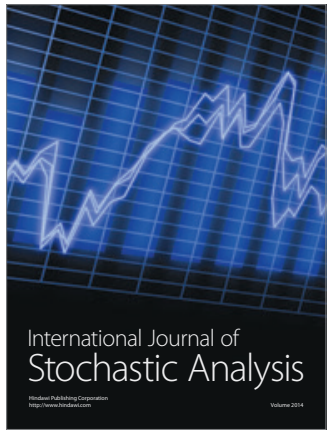

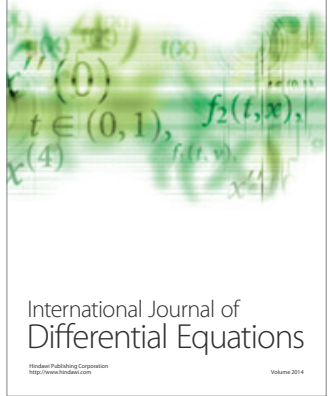
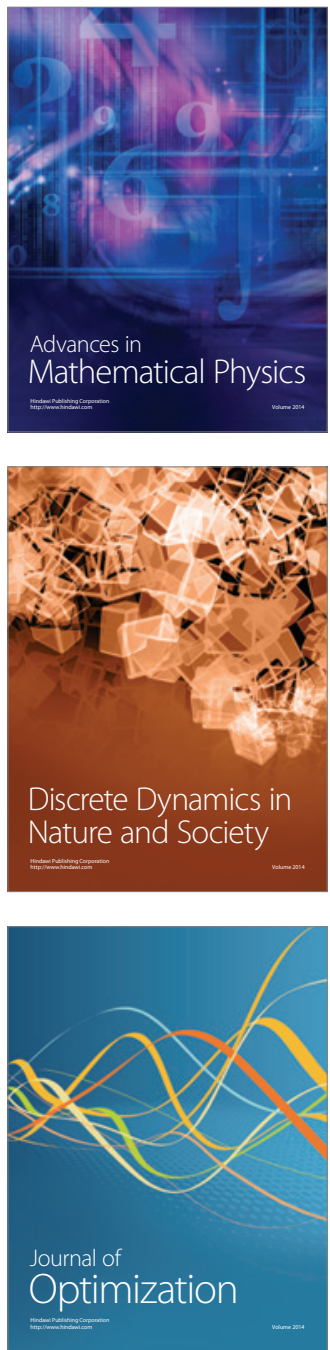